\title{
MEDIDAS PROTETIVAS EM SAÚDE MENTAL: RECOMENDAÇÕES AOS SERVIÇOS DE SAÚDE EM TEMPOS DE PANDEMIA
}

\author{
Maria Giovana Borges Saidel' \\ ORCID: 0000-0002-3259-1760 \\ Elizabeth Esperidião" \\ ORCID: 0000-0002-9284-6243 \\ Cristina Maria Douat Loyola"' \\ ORCID: 0000-0003-2824-6531
}

Jeferson Rodrigues'v ORCID: 0000-0002-8612-9088

Rosane Mara Pontes de Oliveirav ORCID: 0000-0002-2183-1423

Alexsandro Barreto Almeida ${ }^{\mathrm{vI}}$ ORCID: 0000-0002-9389-1572

'Faculdade de Enfermagem, Universidade Estadual de Campinas. Campinas, São Paulo, Brasil.

"Faculdade de Enfermagem, Universidade Federal de Goiás. Goiânia, Goiás, Brasil.

'" Universidade Ceuma. São Luiz, Maranhão, Brasil. IPUB, Universidade Federal do Rio de Janeiro. Rio de Janeiro, Rio de Janeiro, Brasil.

Iv Departamento de Enfermagem, Universidade Federal de Santa Catarina. Florianópolis, Santa Catarina, Brasil.

vEscola de Enfermagem Anna Nery. Rio de Janeiro, Rio de Janeiro, Brasil.

${ }^{v}$ Centro Universitário Estácio Brasília e Centro Universitário de Brasília. Brasília, Distrito Federal, Brasil.

Autor Correspondente: Maria Giovana Borges Saidel E-mail:mgsaidel@unicamp.br

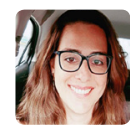

Como citar: Saidel MGB, Esperidião E, Loyola CMD, Rodrigues J, Oliveira RMP, Almeida AB. Medidas protetivas em saúde mental: recomendações aos serviços de saúde em tempos de pandemia. In: Esperidião $E$, Saidel MGB (Orgs.). Enfermagem em saúde mental e COVID-19. 2.ed.rev. Brasília, DF: Editora ABEn; 2020.

p. 17-23. (Série Enfermagem e Pandemias, 4). https://doi.org/10.51234/aben.20.e04.c02

\section{INTRODUÇÃO}

O surto de uma doença infecciosa associado aos diversos medos e incertezas que acompanham uma situação ímpar, sem informações conclusivas, como é o caso da pandemia da COVID-19, pode suscitar diversas emoções negativas e consequentemente levar ao sofrimento psíquico de pessoas independente de quaisquer características pessoais, demográficas ou mesmo epidemiológicas ${ }^{(1)}$. Diante dessa afirmação e da situação crítica da contemporaneidade, trabalhadores de saúde que estão na linha de frente, diretamente envolvidos nos processos de cuidado de pacientes com COVID-19, apresentam risco potencial de desenvolver sintomas de sofrimento psíquico, transtornos mentais ou mesmo sofrer agudizações de transtornos mentais pré-existentes. Esse risco aumentado é associado a diversos fatores, dentre eles, o aumento de casos confirmados cotidianamente e divulgados amplamente pela mídia; carga de trabalho intensa e vários vínculos empregatícios; falta de protocolos específicos para tratamento que apresentem evidências científicas, inadequações e falta de equipamentos de proteção individual (EPI's) ${ }^{(2)}$. Para fins de comparação, alguns estudos descreveram reações psicológicas adversas em 2003 no surto de SARS (Severe Acute Respiratory Syndrome) $)^{(3-5)}$. Os profissionais de saúde temiam o contágio e a infecção de seus familiares, amigos e colegas ${ }^{(3)}$ alguns relataram incertezas e estigmatizações por parte da sociedade ${ }^{(3,4)}$, relatos de altos níveis de estresse, sintomas de ansiedade e depressão(5). Nesse sentido, atualmente frente à pandemia da COVID-19, surgem preocupações semelhantes com a saúde mental dos profissionais de saúde.

Um estudo transversal recente com uma amostra de 1257 profissionais de saúde realizados em 34 hospitais em várias regiões da China, primeiro país a enfrentar a pandemia no ano de 2019, avaliou a extensão dos resultados sobre a saúde mental, bem como fatores associados entre 
profissionais de saúde que estavam na linha de frente da pandemia da COVID-19. Os resultados demonstraram uma proporção considerável de profissionais de saúde que relataram sintomas de depressão (50,4\%), ansiedade $(44,6 \%)$, insônia $(34,4 \%)$ e angústia $(71,5 \%)$, e destaca-se que essa proporção foi maior entre as mulheres, especialmente entre as enfermeiras ${ }^{(2)}$.

O enfrentamento da pandemia expôs fragilidades dos países mais afetados em relação ao cuidado com a saúde mental de seus profissionais de saúde. No entanto, é possível refletir sobre experiências sistematizadas que podem auxiliar essa população. Os gestores precisam atentar-se a fatores que podem contribuir, por um lado, para o adoecimento psíquico desses trabalhadores e, por outro lado, possibilitar intervenções precoces e efetivas(6).

Frente à necessidade e à importância da saúde mental dos profissionais de saúde que atuam na linha de frente da pandemia de COVID-19, surgiu a necessidade de refletir esse contexto fundamentado em literaturas nacionais e internacionais sobre medidas protetivas para a saúde mental que podem ser organizadas por gestores e profissionais de educação permanente dos serviços de saúde no Brasil.

\section{OBJETIVO}

Refletir medidas protetivas em saúde mental para trabalhadores da saúde direcionadas aos gestores e profissionais da educação permanente em tempos de pandemia da covid-19.

\section{MÉTODOS}

A construção metodológica do presente capítulo deu-se por meio da lógica teórica-reflexiva que aborda medidas e estratégias protetivas para a saúde mental do trabalhador com vistas a alcançar gestores e profissionais de educação permanente e continuada dos serviços de saúde. Dessa forma, as reflexões, discussões e considerações foram categorizada em três grupos: 1) Identificar: diagnóstico situacional dos serviços sobre a saúde mental dos trabalhadores da saúde; 2) Planejar: a organização de grupos de especialistas em saúde mental para construir e organizar estratégias; 3 ) Agir: medidas protetivas de saúde mental aos trabalhadores de saúde.

Esse estudo teórico-reflexivo foi construído com base na leitura crítica de referenciais nacionais, internacionais e principalmente a partir de países que estão em outra fase da pandemia da COVID-19 e discutidos frente ao contexto nacional. O processo consistiu em conferir uma ordem às informações em um potente corpo de conhecimento, com o objetivo de avaliar e sumarizar os dados encontrados.

\section{IDENTIFICAR: DIAGNÓSTICO SITUACIONAL DOS SERVIÇOS SOBRE A SAÚDE MENTAL DOS TRABA- LHADORES DE SAÚDE}

Inicialmente é preciso conhecer o perfil dos profissionais de saúde de cada instituição, pois existem diferenças sociodemográficas, epidemiológicas e pessoais que devem ser levadas em conta para que as estratégias sejam direcionadas para essa população que será acolhida pelas ações de saúde mental. Para essa ação, gestores e/ou profissionais da educação permanente e continuada poderiam construir formulários e/ ou aplicar escalas, já validadas para o Brasil, para serem respondidos online (pelo celular) pelos trabalhadores. Importante ressaltar a importância de garantir o anonimato dos profissionais nesse processo para que estes sintam-se à vontade para fornecer as informações sobre seu estado de saúde mental. Essa ação resultaria em um diagnóstico situacional acerca da saúde mental desses profissionais, em especial, como representam e lidam com a COVID-19 no processo de trabalho.

Corroborando com as informações acima, estudo recente descreve três pontos chaves para iniciar intervenções psicológicas: compreender questões sobre a saúde mental em diferentes populações que são influenciadas pela pandemia da COVID-19; identificar indivíduos que apresentam risco alto de sintomas psíquicos, dentre eles, risco de suicídio, heteroagressão e providenciar ações efetivas para as pessoas que precisam. Nesse mesmo estudo, os 
autores colocam os profissionais de saúde que atuam na linha de frente no nível 1 de vulnerabilidade, ou seja, essa população estaria compondo o grupo mais vulnerável para desenvolver sintomas de sofrimento psíquico(7).

Nessa fase é preciso destacar também a importância em identificar profissionais de saúde com transtornos mentais pré-existentes, pois para esses trabalhadores, os surtos de doenças altamente infecciosas, podem significar um fator de risco para a agudização de algum sintoma e consequentemente o agravamento do transtorno mental ${ }^{(8)}$.

Essa fase é fundamental, pois para as estratégias e medidas protetivas serem realmente efetivas, elas terão que ter acurácia e serem precisas. Nesse sentido, quanto mais essas medidas forem direcionadas para o público alvo pretendido, considerando-se as diferenças culturais, sociodemográficas e epidemiológicas, maior a probabilidade de serem adequadas. Portanto, é preciso considerar as diferenças que temos em nosso país, nas macro e microrregiões em especial os diferentes processos de trabalho, organização de trabalho, conflitos nas relações já existentes, a maneira como cada profissional se relaciona com os usuários do sistema de saúde, a condição de cada local de trabalho e a forma como cada gestor induz estratégias para lidar com o cotidiano, que foi e está agravado com a pandemia. Esse cuidado com as diferenças é primordial para que haja um diagnóstico mais fidedigno sobre a saúde mental dos trabalhadores.

\section{PLANEJAR: ORGANIZAÇÃO DE GRUPOS DE ESPECIALISTAS EM SAÚDE MENTAL PARA EDIFICAR E ORGANIZAR ESTRATÉGIAS}

Logo após a realização do diagnóstico situacional, será preciso sistematizar o planejamento das futuras estratégias que terão como objetivo primordial a construção das ações para proteção da saúde mental e acolhimento dos sentimentos de sofrimento psíquico desses trabalhadores. Nesse sentido, a organização de um grupo de trabalho com profissionais com formação específica voltada para questões da saúde mental e trabalho é fundamental.

Salienta-se a importância de uma equipe multidisciplinar de saúde mental (enfermeiros especialistas em saúde mental, médicos psiquiatras e psicólogos) na composição deste grupo, além de serem pessoas que conhecem a estrutura da organização e têm habilidade e competência para implementar, executar e avaliar as ações que serão planejadas nessa fase.

Nesse sentido, há uma compreensão de que esses profissionais com expertise inerente à sua formação e detentores de experiência com a saúde mental poderão atuar a partir das relações entre os sujeitos desenvolvendo mais a intersubjetividade, fortalecendo recursos para lidar com as adversidades ${ }^{(9)}$. A formação em saúde mental desse grupo de especialistas, possibilitaria uma ampliação da compreensão da singularidade dos indivíduos que apresentam risco e/ou sintomas de sofrimento psíquico, no caso, os profissionais de saúde. Nesse sentido, a formação deste grupo de trabalho deve incluir um vasto arsenal de atuações e intervenções clínicas e também contemplar aspectos que permitam maior compreensão e possibilidades de posicionamento nas relações institucionais ${ }^{(10)}$.

Esse grupo de especialistas também foi considerado em documento elaborado pelo Centro de Documentação para Promoção da Saúde na Itália, em decorrência da pandemia da COVID-19. O documento descreve que o grupo de especialistas deveria se debruçar também sobre iniciativas educacionais e de aprendizagem em saúde mental durante todo o processo da pandemia ${ }^{(11)}$.

Portanto, esse grupo teria a responsabilidade de se debruçar sobre os resultados do diagnóstico situacional em saúde mental dos profissionais de saúde, para que estes subsidiem as discussões do planejamento e construção de ações que sejam efetivas para aquela população. As problematizações emergidas desse grupo de profissionais sobre os processos de trabalho dos equipamentos de saúde, devem ser pautadas da perspectiva da Educação Permanente (EP). Essa lógica possibilita reflexões sobre os processos de trabalho como uma estratégia de reorganização das práticas educativas como ação para futuras ofertas clínicas que teriam fundamentação importante para medidas protetivas futuras. 


\section{AGIR: MEDIDAS PROTETIVAS DE SAÚDE MENTAL AOS TRABALHADORES DE SAÚDE}

Nesse item serão abordadas algumas medidas protetivas implementadas em países que já passaram pela fase aguda da pandemia e/ou encontram-se em um outro momento. Um ponto de destaque são as adaptações necessárias frente às realidades de nosso contexto nacional que poderão ser construídas no grupo de trabalho dos profissionais de saúde mental. Todas as medidas descritas podem contribuir para prevenção do adoecimento psíquico dos trabalhadores de saúde, promovendo a saúde mental; prevenir a agudização dos sintomas de transtornos mentais pré-existentes e reduzir o risco de estresse pós-traumático desses trabalhadores após a pandemia da COVID-19.

O uso de tecnologia digital (plataforma de internet e smartphones) para as intervenções tem sido descrita com frequência, com destaque para a presença de equipes multiprofissionais. Essas intervenções são recomendadas em virtude dos resultados significativos. As características descritas como eficazes nessa medida de atendimentos em saúde mental por meio de plataformas digitais são: dinamismo e oportunidade além do desenvolvimento de fases para serem pensadas (durante e após a pandemia). Destaca-se a importância de treinamentos específicos da equipe multidisciplinar e de supervisão clínica constante dos profissionais após os atendimentos ${ }^{(7,12-13)}$. A realização de atendimentos em saúde mental, por meio telefônico, também tem sido descrita como eficaz nesse momento da pandemia. Uma revisão conduzida em 2011 sobre psicoterapia fornecida por telefone, por meio de análise de ensaios randomizados, aborda a temática descrevendo os benefícios e desafios para esse tipo de atendimento ${ }^{(14)}$.

É importante o oferecimento de treinamento/capacitações sobre intervenções em situações de crise de maneira sistemática, demonstrar aos profissionais de saúde como identificar as respostas do estresse, bem como aprender possiblidades de conduzir emoções no decorrer dos processos de trabalho ${ }^{(11)}$. Aqui no Brasil, no início da pandemia, vários hospitais 'correram contra o tempo' para organizar capacitações referentes ao uso de EPI's, intubação de pacientes com COVID entre outros processos de trabalho.

Conforme indica essa referência italiana ${ }^{(11)}$, essas capacitações deveriam compreender também ações de saúde mental. Nesse sentido, essas capacitações poderiam incluir possibilidades e estratégias para o cuidado e autocuidado em saúde mental (técnicas de relaxamento, meditação, respiração, contemplação; estratégias de autocuidado no cotidiano, identificação de sintomas de sofrimentos psíquico e como buscar ajuda, entre outras).

Condução de entrevistas individuais e discussões em grupo sobre sentimentos internos, possibilidade de suporte e acolhimento, mobilização de recursos internos e preparação para os momentos de estresse ${ }^{(11)}$. Essas entrevistas poderiam ser conduzidas por meio do uso de tecnologia remota ou mesmo presencialmente, considerando-se todos os cuidados de segurança para os envolvidos no contexto de atendimento, obedecendo, o distanciamento e o uso de EPI's.

Sublinha-se, também, as estratégias ineficazes para conduzir emoções negativas, como o uso de substâncias psicoativas, pois no longo prazo essas substâncias podem contribuir para o agravamento de quadros de sofrimento humano e de transtornos mentais ${ }^{(11)}$.

Ofertar informações de qualidade para os profissionais, criar e alimentar canais oficiais (internos) de comunicação e atualização sobre a pandemia, evitando assim, as notícias falsas e alarmistas, pois a gestão da informação é fundamental para que não ocorra excesso de busca e fake news. Essas medidas são descritas como um atenuante das preocupações e incertezas dos trabalhadores, além de oferecer uma sensação de direcionamento sobre a situação real ${ }^{(11,12)}$.

Outras medidas protetivas da saúde mental destacadas por outros países são: possibilidades de momentos de descanso dos trabalhadores em locais adequados após óbitos ou más notícias ${ }^{(11)}$; treinamento para profissionais in loco para a identificação precoce de profissionais que precisem de intervenção, com a garantia de confidencialidade e espaço adequado para que possam externalizar os sentimentos de sofrimento psíquico ${ }^{(8,11-12)}$; disponibilidade de um 'pronto socorro' para atender essas demandas dos profissionais de saúde nas instituições, a disponibilidade de profissionais da saúde mental para atendimento imediato em casos de crises e/ou situação que precisem de intervenção imediata ${ }^{(11)}$. 
Importante salientar que as medidas protetivas devem sempre serem analisadas através de uma perspectiva do público especifico, pois, o enfrentamento dos sofrimentos não possui a mesma representação para cada pessoa, ou seja, as ações devem ser analisadas de forma complexa. Mas devem ser implantadas respeitando os diferentes fatores da realidade do local e principalmente da singularidade dos sujeitos, para que se tenha resultados positivos.

O grande desafio do profissional da saúde para prevenção do adoecimento psíquico e promover a saúde mental é ter como base de enfrentamento da pandemia, princípios que privilegiam a construção do conhecimento, o aprendizado significativo, interdisciplinar e integrador do pensamento racional, estético, ético e humanista. O foco deverá estar na aprendizagem de um novo olhar para um novo natural que não privilegia mais o contato corpo a corpo, mas nos oferta a tecnologia como um instrumento de amplas possibilidades ${ }^{(15)}$. Logo, o custo financeiro destas estratégias de intervenção deve ser visto como investimento no capital humano e na perspectiva de benefício a saúde do trabalhador.

\section{Limitações do Estudo}

O presente capítulo traz uma descrição fundamentada na lógica reflexiva após análises da literatura nacional e internacional, essa metodologia apontou as medidas em sua maioria pautadas em contextos internacionais. Nesse sentido, é preciso estudos quantitativos e qualitativos em território nacional para que profissionais de saúde brasileiros possam participar e pesquisadores possam avaliar os impactos da pandemia na saúde mental desses trabalhadores.

\section{Contribuições para a Área}

Acredita-se que o texto contribui em dois aspectos para a área de saúde mental de profissionais de saúde frente a COVID-19. A primeira, é que a partir das experiências de outros países que estão em outro momento da pandemia, é possível aprender com exitosas, passíveis de avaliação e considerar possibilidades de adaptação em contexto nacional. A outra, é a possibilidade, após reflexões dessas medidas, viabilizar a operacionalização nos serviços de saúde por gestores e setor de educação continuada. Nesse sentido, o cuidado da saúde mental dos profissionais de saúde, que hoje estão na linha de frente é possível e fundamental, e as medidas apresentadas mostram um caminho possível.

\section{CONSIDERAÇÕES FINAIS}

A situação de globalização do mundo e de rapidez de movimentação é propícia a pandemias e há possibilidade de que elas se repitam neste século. Há que se relevar e atentar para a máxima de que não existe saúde sem saúde mental. Os transtornos mentais, bem como sintomas de sofrimento psíquico tem potência para produzir imperícia e absenteísmo no trabalho. Precisamos ter equipes treinadas no tema da saúde mental e nos meios de comunicação virtual para contato individualizado, destacando-se o uso de tecnologias digitais. Estas equipes precisam ter a sofisticação de alcançar a singularidade das pessoas em sofrimento, reconhecendo as diferenças regionais e sócio econômicas e possuir também conhecimento operacional e institucional sobre os locais de trabalho. É preciso haver locais adequados e esteticamente interessantes e harmônicos para descanso dos profissionais de modo a garantir momentos de estresse mais baixo na linha de frente de trabalho. As equipes de saúde mental devem ser capazes de identificar precocemente as respostas ineficazes ao estresse, ressaltando-se nesse aspecto o uso de substâncias psicoativas e a automedicação. Também é interessante propor alguma seleção e proteção contra a velocidade das informações e sobretudo sobre as fakenews. Destarte, este capitulo ao refletir medidas protetivas e promotoras de saúde mental aos trabalhadores em tempos da COVID-19, busca contribuir com a sensibilização de gestores do setor da saúde para que institucionalizem 
estratégias para os trabalhadores terem mais recursos para lidar com o contexto atual e até mesmo para situações semelhantes no futuro.

Frente a isso, importante considerar o papel fundamental de gestores e profissionais de educação continuada nas implementações de tais medidas como forma de demonstrar que a estrutura organizacional dos serviços de saúde pode contribuir para o cuidado em saúde mental dos profissionais de saúde.

\title{
AGRADECIMENTO
}

\author{
Ao Departamento de Enfermagem Psiquiátrica e Saúde Mental/Associação Brasileira de Enfermagem \\ (DEPSM/ABEn)
}

\section{REFERÊNCIAS}

1. Ho CS, Chee CY, Ho RC. Mental Health Strategies to Combat the Psychological Impact of COVID-19 Beyond Paranoia and Panic. Ann Acad Med Singapore. [Internet] 2020 [cited 2020 mai 30];49(3):155-160. Available from: https://pubmed.ncbi. nlm.nih.gov/32200399/

2. Lai J, Ma S, Wang Y, et al. Factors Associated With Mental Health Outcomes Among Health Care Workers Exposed to Coronavirus Disease 2019. JAMA Netw Open. [Internet] 2020 [cited 2020 mai 30];3(3):e203976. Available from: https:// jamanetwork.com/journals/jamanetworkopen/fullarticle/2763229

3. Maunder R, Hunter J, Vincent L, et al. The immediate psychological and occupational impact of the 2003 SARS outbreak in a teaching hospital. CMAJ. [Internet] 2003 [cited 2020 jun 05];168(10). Available from: https://www.ncbi.nlm.nih.gov/pmc/ articles/PMC154178/

4. Bai Y, Lin CC, Lin CY, Chen JY, Chue CM, Chou P. Survey of stress reactions among health care workers involved with the SARS outbreak. Psychiatr Serv. [Internet] 2004 [cited 2020 jun 06];55(9):1055-1057.Available from: https://pubmed.ncbi. nlm.nih.gov/15345768/

5. Lee AM, Wong JG, McAlonan GM, et al. Stress and psychological distress among SARS survivors 1 year after the outbreak. Can J Psychiatry. [Internet] 2007 [cited 2020 jun 06];52(4):233-240. Available from: https://pubmed.ncbi.nlm.nih.gov/17500304/

6. Saidel MGB, Lima MHM, et al. Campos CJG. Intervenções em saúde mental para profissionais de saúde frente a pandemia de Coronavírus. Rev enferm UERJ. [Internet] 2020 [cited 2020 jun 15];28:e49923. Available from: https://www.epublicacoes.uerj.br/index.php/enfermagemuerj/article/view/49923

7. Li W, Yang Y, Liu ZH, Zhao YJ, Zhang Q, Zhang L, Cheung T, Xiang YT. Progression of Mental Health Services during the COVID-19 Outbreak in China. Int J Biol Sci [Internet] 2020 [cited 2020 jun 15];16(10):1732-1738. Available from: https:// www.ijbs.com/v16p1732.htm

8. Hall RCW, Hall RCW, Chapman MJ. The 1995 Kikwit Ebola outbreak: lessons hospitals and physicians can apply to future viral epidemics. Gen Hosp Psychiatry. [Internet] 2008 [cited jun 28];30:446-52. Availble from: https://www.ncbi.nlm.nih. gov/pmc/articles/PMC7132410/

9. Schran LS, Machineski GG, Rizzotto, MLF, et al. Percepção da equipe multidisciplinar sobre a estrutura dos serviços de saúde mental: estudo fenomenológico. Rev Gaúcha Enferm. [Internet] 2019 [cited jun 28];40,e20180151. Available from: https://www.scielo.br/scielo.php?script=sci_arttext\&pid=S1983-14472019000100407\&lang=pt

10. Emerich F, Onocko-Campos R. Formação para o trabalho em Saúde Mental: reflexões a partir das concepções de Sujeito, Coletivo e Instituição. Interface - Comunicação, Saúde, Educação. [Internet] 2019 [cited 2020 jun 28];23,e170521. Available from: https://www.scielo.br/scielo.php?script=sci_abstract\&pid=S1414-32832019000100212\&lng=en\&nrm=iso\&tlng=pt

11. Longo R, Penasso M, Scarponi S (ed). La salute mentale degli operatori sanitari durante e dopo la pandemia di COVID 19: Quali le evidenze e le linee guida per gli interventi di supporto psicológico e psicosociale. Centro di Documentazione per la Promozione della Salute (DoRS). Regione Piemonte. [Internet] 2020 [cited 2020 jun 29]. Available from: https://www. dors.it/documentazione/testo/202005/Salute\%20mentale\%20operatori\%20sanitari\%20-\%20200508.pdf

12. Jun Z, Weili W, Xin Z, et al. Recommended psychological crisis intervention response to the 2019 novel coronavirus pneumonia outbreak in China: a model of West China Hospital. Precision Clinical Medicine. [Internet] 2020 [cited 2020 jun 29];3(1),3-8. Available from: https://www.ncbi.nlm.nih.gov/pmc/articles/PMC7107095/ 
13. Wang C, Pan R, Wan X et al. Immediate psychological responses and associated factors during the initial stage of Coronavirus Disease (COVID-19) epidemic among the general population in China. Int J Environ Res Public Health [Internet] 2020 [cited 2020 jun 29];17(5),1729. Available from: https://www.ncbi.nlm.nih.gov/pmc/articles/PMC7084952/

14. Brenes GA, Ingram CW, Danhauser SC. Benefits and challenges of conducting psychotherapy by telephone. Prof Psychol Res Pr. [Internet] 2011 [cited 2020 jun 29];42,543-549. Available from: https:/www.ncbi.nlm.nih.gov/pmc/articles/ PMC3256923/

15. Moran JM. A educação que desejamos: Novos desafios e como chegar lá. Papirus, 2007. 